\title{
A Descriptive Case Series of Patients with End-Stage Liver Disease Hospitalized with Variceal Bleeding Who Received Pharmacologic Venous Thromboembolism Prophylaxis
}

\author{
Anneliese M. Schleyer, MD, MHA ${ }^{1}$ \\ Mark W. Smith, MD ${ }^{1}$ \\ Kenneth M. Jarman, PharmD ${ }^{2}$ \\ Paul K. Crane, $\mathrm{MD} \mathrm{MPH}^{1}$ \\ Astrid B. Schreuder, $\mathrm{PhD}^{3}$ \\ J. Richard Goss, MD $\mathrm{MPH}^{1,3}$
}

\author{
${ }^{1}$ Division of General Internal Medicine, Department of Medicine, Harborview Medical Center, Seattle, \\ Washington. \\ ${ }^{2}$ Department of Pharmacy, Harborview Medical Center, Seattle, Washington. \\ ${ }^{3}$ Department of Quality Improvement, Harborview Medical Center, Seattle, Washington.
}

Disclosure: Nothing to report.

BACKGROUND: It is unknown whether venous thromboembolism prophylaxis (VTEP) should be utilized in hospitalized patients with end-stage liver disease (ESLD), particularly in those admitted with variceal bleeding. OBJECTIVE: We sought to describe a cohort of patients who received pharmacologic VTEP, specifically identifying the occurrence of rebleeding.

DESIGN: Descriptive case series.

SETTING/PATIENTS: All adult patients with ESLD admitted to an urban county teaching hospital over three years with variceal bleeding who received pharmacologic VTEP during hospitalization.

RESULTS: A total of 22 patients with ESLD and variceal bleeding received pharmacologic VTEP. Only 1 patient rebled after initiation of VTEP; 2 patients were diagnosed with lower extremity deep venous thrombosis while on VTEP including the 1 patient who rebled.

CONCLUSIONS: VTEP was associated with an unexpectedly low incidence of recurrent bleeding in patients with ESLD and variceal bleeding. Further study may be warranted. Journal of Hospital Medicine 2011;6:151-155. @ 2010 Society of Hospital Medicine.

\section{KEYWORDS: liver disease, thromboembolism prophylaxis, variceal bleeding.}

Additional Supporting Information may be found in the online version of this article.

Venous thromboembolism (VTE) is a major cause of morbidity and mortality in hospitalized patients. ${ }^{1-3}$ Major efforts are underway to increase appropriate VTE prophylaxis $(\text { VTEP })^{4}$ and adherence to VTEP guidelines are increasingly used as a quality of care measure. National 2008 VTEP guidelines suggest that all medical patients ill enough to require hospitalization, particularly those requiring admission to the Intensive Care Unit (ICU), have at least a moderate risk of developing VTE and prophylaxis is recommended. ${ }^{4}$ Hospitalized patients with end-stage liver disease (ESLD), despite their coagulopathy, are known to be at risk for $\mathrm{VTE}^{4-8}$ and may be VTEP candidates.

Based on available literature, it is unknown whether pharmacologic VTEP should be utilized in acutely ill, hospitalized patients with ESLD, particularly in those admitted with variceal bleeding. These patients are at high risk for rebleeding, with the highest risk in the first 5 days. ${ }^{9}$ Early rebleeding, defined as recurrent bleeding within 6 weeks of initial bleed, declined from $47 \%$ in the 1980 s to $13 \%$ by 2000 because of increased early endoscopic intervention and use of medications to prevent rebleeding. ${ }^{9-11}$ In multicenter cohort studies, D'Amico and De Franchis ${ }^{12}$ reported that $13 \%$ of patients with variceal bleeding had uncontrolled bleeding, rebleeding, or death within 5 days of admission while Bahmba et al. ${ }^{13}$ reported a $16 \%$ rate of rebleeding within 5 days. We are unaware of prior reports regarding the safety of VTEP in this high-risk group of patients.

\section{Objective}

We sought to describe rebleeding in a series of 22 patients with ESLD admitted with variceal bleeding who received pharmacologic VTEP.

\section{Methods}

We identified all patients 18 years and older with upper gastrointestinal bleeding admitted to Harborview Medical Center, a 400-bed urban county teaching hospital in Seattle, Washington, between January 1, 2003 and December 31, 2005 (Figure 1), just prior to medical center-wide implementation of a VTEP guideline. Potential cases were identified using administrative data based on 8 discharge diagnoses (Supporting Information Appendix 1) and 10 procedure codes (Supporting Information Appendix 2). ${ }^{14}$ Inpatient pharmacy data indicating continuous octreotide infusion were used to refine the sample. At our institution, it is a standard of care to 


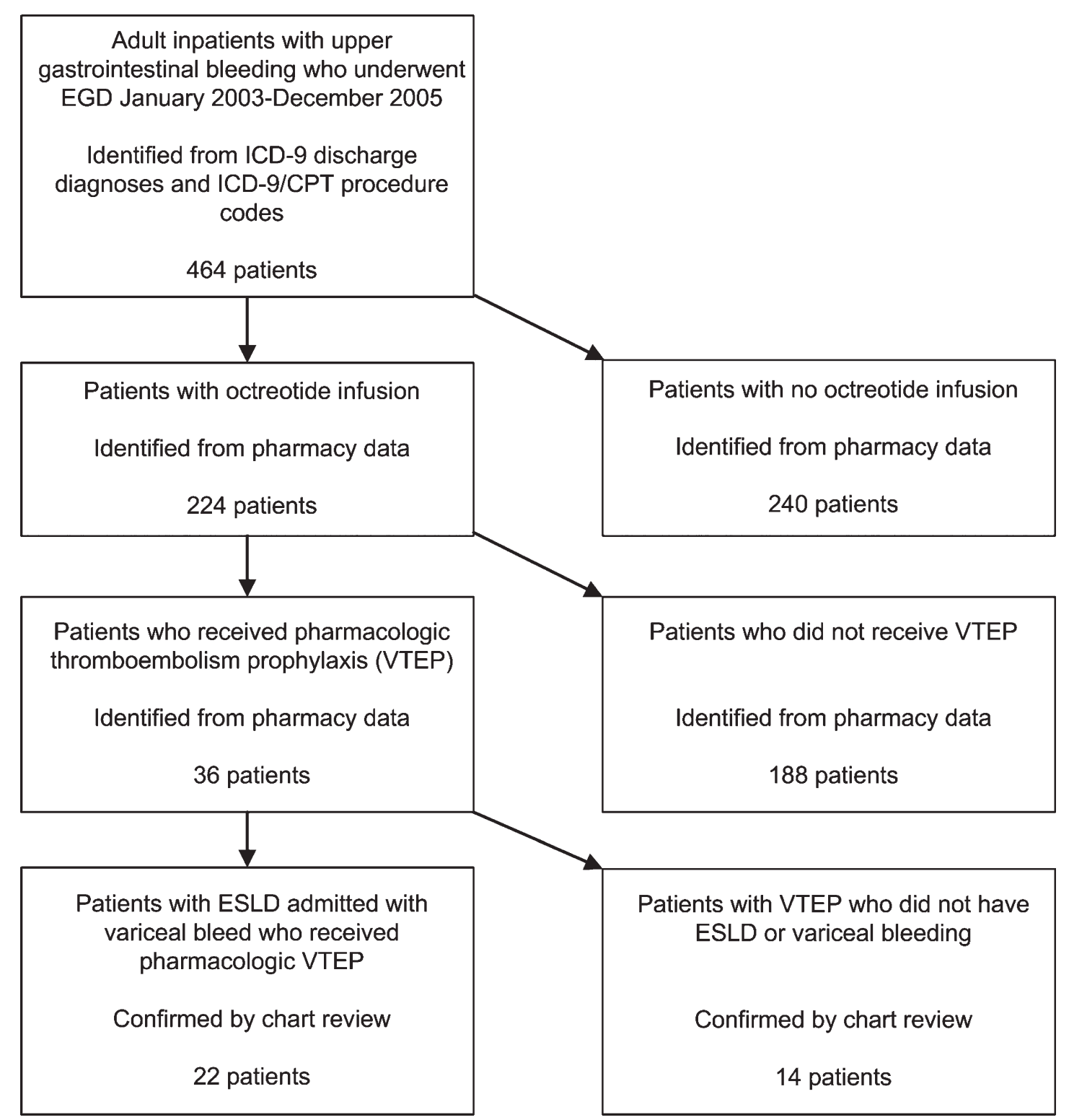

FIGURE 1. Patient identification: Patients with end-stage liver disease (ESLD) admitted with variceal bleed who received pharmacologic venous thromboembolism prophylaxis. EGD, esophagogastroduodenoscopy; ICD-9, International Classification of Disease, Version 9 diagnosis codes; CPT, common procedural terminology (CPT) procedure codes; VTEP, venous thromboembolism prophylaxis.

initiate octreotide in patients admitted with variceal bleeding. We excluded patients who did not have ESLD (defined as evidence of cirrhosis and associated complications including but not limited to ascites, encephalopathy, variceal bleeding, portal hypertension) documented in their problem list or past medical history and those with no variceal bleeding based on medical record review. We identified cases receiving pharmacologic VTEP, either subcutaneous unfractionated heparin (UFH) or low molecular weight heparin (LMWH), during hospitalization from pharmacy records.

We obtained demographic and clinical data from administrative billing systems, electronic and paper medical records, and inpatient pharmacy databases and verified transfusion data from the Puget Sound Blood Center. We abstracted esophagogastroduodenoscopy (EGD) findings indicating high risk of rebleeding including variceal grade and stigmata of recent bleeding such as red spots or wales. ${ }^{15,16}$ Data were abstracted by the first 3 authors (AS, MS, KJ) and reviewed again by 2 authors (AS, KJ) blinded to the others' abstractions.

We calculated Model for ESLD (MELD) scores on admission. These scores correlate with 3 month mortality in ESLD. ${ }^{17}$ We tabulated 5 factors shown in some studies to predict bleeding including high International Normalized 
TABLE 1. Characteristics of Hospitalized Patients with End-Stage Liver Disease and Variceal Bleeding Who Received Pharmacologic Thromboembolism Prophylaxis $(n=22)$

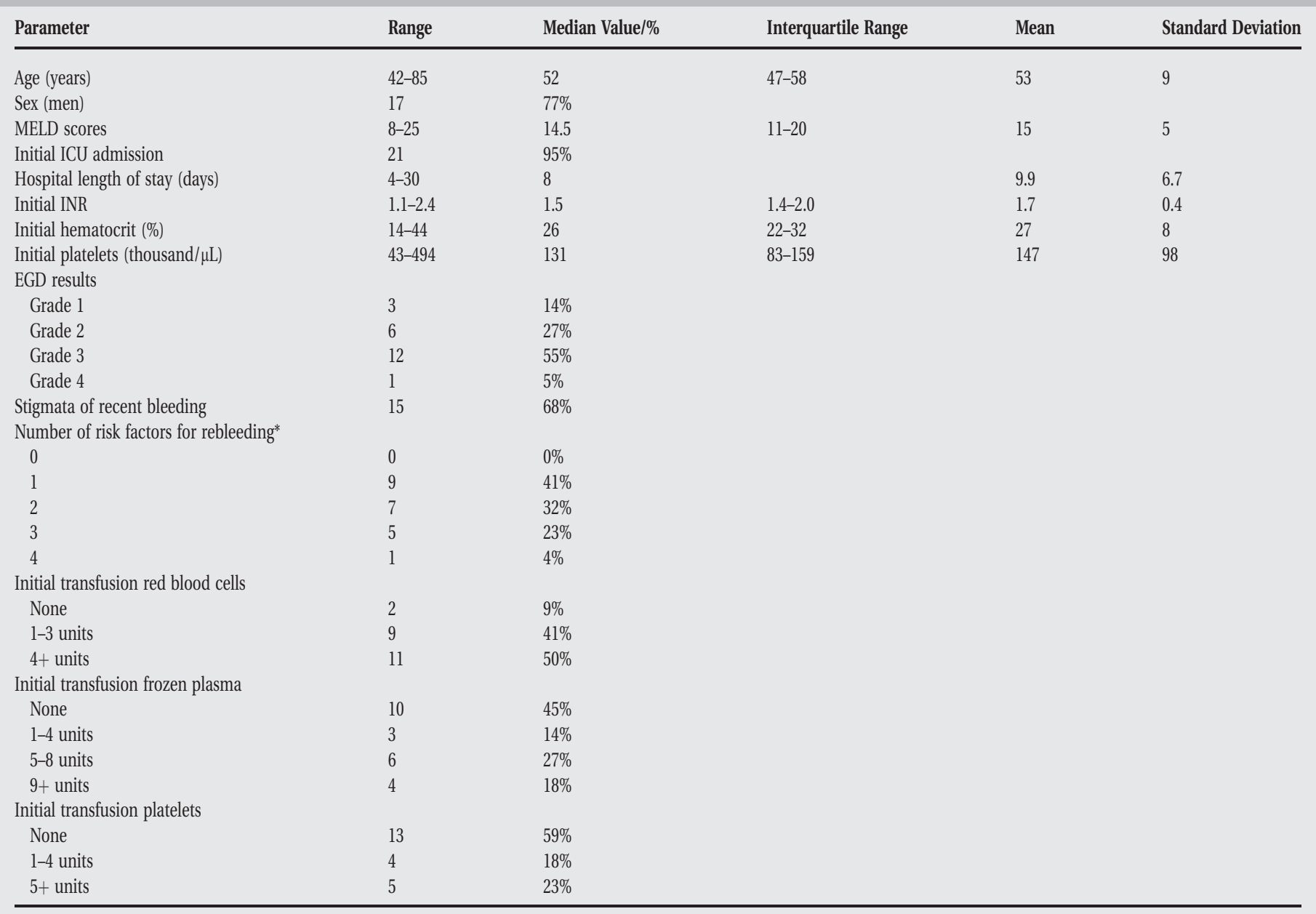

Ratio (INR) (>1.5), low hematocrit $(<25 \%)$, low platelet count $(<100,000$ per microliter $)$, active bleeding at EGD, and transfusion of four or more units of red cells within 24 hours of admission. ${ }^{10-13}$

We defined rebleeding as a decrease in hematocrit of greater than 5 percentage points compared with postresuscitation hematocrit, transfusion of additional red cells more than 48 hours after initial resuscitation, repeat unscheduled EGD, or return to the ICU for therapies related to rebleeding. ${ }^{18}$ The University of Washington Human Subjects Board approved this study.

\section{Results}

Of 224 patients initially identified, 36 received pharmacologic VTEP. We excluded 14 who did not have ESLD $(n=1)$ or did not have a variceal bleed $(n=13)$. The remaining 22 patients form the sample described in Figure 1.
The median age of patients was 52 years (range 42-85) and $77 \%$ were men (Table 1). Twenty-one of 22 patients (95\%) were initially admitted to the ICU; median length of stay was 8 days (range 4-30). Median MELD score on admission was 15 (range 8-25). On EGD, the number of variceal columns ranged from 1 to 4 ; 17 patients (77\%) had at least 3 . A total of 15 patients (68\%) had stigmata of recent bleeding and $16(72 \%)$ underwent banding (range 1-6 bands). All patients had at least 1 bleeding risk factor (Table 1) of which the most common factors observed were initial transfusion of 4 or more units of red cells $(50 \%, n=11)$, INR $>1.5(45 \%$, $\mathrm{n}=10)$, and hematocrit $<25 \%(45 \%, \mathrm{n}=10)$.

A total of 12 patients (55\%) received 5000 units of UFH every 8 hours, 8 (36\%) received 5000 units UFH every 12 hours, and $2(9 \%)$ received LMWH. VTEP was initiated as early as day of admission and as late as day 19. Median VTEP start date was hospital day 4. Median duration of of VTEP was 5 days. 
Only 1 patient $(4.5 \%)$ rebled after VTEP initiation. The patient received UFH every 8 hours starting on hospital day 6 , and rebleeding occurred on day 9. Repeat EGD showed ulcers at banding sites. The patient was restarted on VTEP on hospital day 13 without recurrence of rebleeding. This patient had a MELD score of 24, initial INR $>2$, hematocrit $<25 \%$, had grade 3 varices and stigmata of recent bleeding on EGD, and received 4 units of packed red cells. These values are similar to those of the cohort as a whole (Table 1). This patient also was diagnosed with DVT while receiving VTEP on hospital day 15. This patient's coagulopathy was in the setting of terminal illness; the patient expired on hospital day 25.

One additional patient rebled prior to VTEP initiation on day 3 with repeat EGD showing a bleeding varix. This patient was nevertheless started on VTEP 4 days after rebleeding. Despite use of VTEP, this patient was diagnosed with DVT on hospital day 9 (and may well have had the DVT at the time of VTEP initiation). The patient was transitioned to therapeutic dose heparin which was tolerated without recurrence of rebleeding.

There were no other confirmed cases of DVT in this series. One additional patient underwent angiogram that showed no pulmonary embolism; 2 other patients underwent lower extremity ultrasounds that were negative for DVT.

\section{Discussion}

At our medical center, only a few inpatients with ESLD admitted with variceal bleed received VTEP. These patients were seemingly at high risk for bleeding and rebleeding given high MELD scores, variceal bleeding, and presence of at least one clinical factor suggesting bleeding risk, and in several cases 3 or more such factors. ${ }^{13,18}$ Despite this, only 1 patient rebled while receiving VTEP. We captured rebleeding rates only during the index hospitalization. We therefore may underestimate early rebleeding rates. ${ }^{10-13}$ Nevertheless, our inpatient data included complete coverage of the earliest period after the index bleeds and the period during which patients were exposed to VTEP, which should be the time of highest rebleeding risk related to VTEP exposure. Interestingly the patient who rebled while on VTEP was also diagnosed with VTE while on VTEP. Two patients $(9 \%)$ in our sample were diagnosed with VTE.

This case series is limited by its small sample size, retrospective nature, single center observation, and perhaps especially by possible selection bias. We were unable to specifically quantify rebleeding risk. Several authors have identified individual factors associated with rebleeding, ${ }^{10-13}$ these were tabulated for patients in this case series (Table 1) and all patients had at least 1 of these factors. Concurrent infection and hepatic vein pressure gradient have been shown to predict rebleeding; ${ }^{9,19}$ we were unable to identify these factors in our data.
There was considerable variability in this case series in timing of VTEP initiation relative to initial bleed. We were unable to characterize provider or patient characteristics that may have influenced the decision to initiate VTEP and timing. The sample size was also too small to comment upon factors associated with choice of UFH versus LMWH and any potential differences in rebleeding risk between the 2. We also did not look at outcomes postindex hospitalization so we can not comment on the extended risk of rebleeding with VTEP after discharge. However, the risk of rebleeding is highest within the first 96 hours $^{13}$ and all patients in this series were hospitalized at least 4 days. Nonetheless, we captured all patients with ESLD and variceal bleeding exposed to VTEP at a large center over a three-year period and found rebleeding rates less than what might be expected.

\section{Conclusions}

Our observations suggest that some inpatients with ESLD and variceal bleeding may tolerate pharmacologic VTEP. In this small group of patients, VTEP was associated with an unexpectedly low incidence of rebleeding. While this case series does not support broad use of VTEP in this population, the lower-than-expected rates of rebleeding suggest that further study of the safety and effectiveness of pharmacologic VTEP in inpatient populations with ESLD may be warranted, particularly given the recommendations of recent national VTE prophylaxis guidelines. ${ }^{4}$

\section{Address for correspondence and reprint requests:}

Anneliese M. Schleyer, MD, MHA, Harborview Medical Center, 325 Ninth Avenue, Box 359780, Seattle, Washington, 98104;

Telephone: 206-744-3000; Fax: 206-744-6063;

E-mail: schleyer@u.washington.edu Received 16 October 2009;

revision received 12 February 2010; accepted 15 March 2010.

\section{References}

1. Aujesky D, Roy PM, Le Manach CP, et al. Validation of a model to predict adverse outcomes in patients with pulmonary embolism. Eur Heart $J$. 2006;27(4):476-481.

2. White RH. The epidemiology of venous thromboembolism. Circulation. 2003;107(23 Suppl 1):I4-I8.

3. Anderson FA Jr, Wheeler HB, Goldberg RJ, Hosmer DW, Forcier A. The prevalence of risk factors for venous thromboembolism among hospital patients. Arch Intern Med. 1992;152(8):1660-1664.

4. Geerts WH, Bergqvist D, Pineo GF, et al. Prevention of venous thromboembolism: American College of Chest Physicians Evidence-Based Clinical Practice Guidelines. 8th Edition. Chest. 2008;133(6 Suppl):381S-453S.

5. Northup PG, McMahon MM, Ruhl AP, et al. Coagulopathy does not fully protect hospitalized cirrhosis patients from peripheral venous thromboembolism. Am J Gastroenterol. 2006;101(7):1524-1528; quiz 680.

6. Amitrano L, Guardascione MA, Brancaccio V, Balzano A. Coagulation disorders in liver disease. Semin Liver Dis. 2002;22(1):83-96.

7. Gulley D, Teal E, Suvannasankha A, Chalasani N, Liangpunsakul S. Deep vein thrombosis and pulmonary embolism in cirrhosis patients. Dig Dis Sci. 2008;53(11):3012-3017.

8. Sogaard KK, Horvath-Puho E, Gronbaek H, Jepsen P, Vilstrup H, Sorensen HT. Risk of venous thromboembolism in patients with liver disease: a 
nationwide population-based case-control study. Am J Gastroenterol. 2009;104(1):96-101.

9. de Franchis R, Dell'Era A. Non-invasive diagnosis of cirrhosis and the natural history of its complications. Best Pract Res Clin Gastroenterol. 2007;21(1):3-18.

10. Chalasani N, Kahi C, Francois F,et al. Improved patient survival after acute variceal bleeding: a multicenter, cohort study. Am J Gastroenterol. 2003;98(3):653-659.

11. Carbonell N, Pauwels A, Serfaty L, Fourdan O, Levy VG, Poupon R. Improved survival after variceal bleeding in patients with cirrhosis over the past two decades. Hepatology. 2004;40(3):652-659.

12. D'Amico G, De Franchis R. Upper digestive bleeding in cirrhosis. Posttherapeutic outcome and prognostic indicators. Hepatology. 2003;38(3): 599-612.

13. Bambha K, Kim WR, Pedersen R, Bida JP, Kremers WK, Kamath PS. Predictors of early re-bleeding and mortality after acute variceal haemorrhage in patients with cirrhosis. Gut. 2008;57(6):814-820.
14. Smith MW, Jarma KM, Schleyer AM, Schreuder AB, Goss R, Onstad S. Use of hospital administrative data to assess quality improvement initiatives. J Gen Intern Med. 2007;22(Supplement).

15. Jalan R, Hayes PC. UK guidelines on the management of variceal haemorrhage in cirrhotic patients. Gut. 2000,2000;46(90003):iii1-15.

16. Siringo S, McCormick PA, Mistry P, Kaye G, McIntyre N, Burroughs AK. Prognostic significance of the white nipple sign in variceal bleeding. Gastrointest Endosc. 1991;37(1):51-55.

17. Kamath PS, Wiesner RH, Malinchoc $\mathrm{M}$, et al. A model to predict survival in patients with end-stage liver disease. Hepatology. 2001;33(2): 464-470.

18. de Franchis R. Evolving consensus in portal hypertension. Report of the Baveno IV consensus workshop on methodology of diagnosis and therapy in portal hypertension. J Hepatol. 2005;43(1):167-176.

19. Hou MC, Lin HC, Liu TT, et al. Antibiotic prophylaxis after endoscopic therapy prevents rebleeding in acute variceal hemorrhage: a randomized trial. Hepatology. 2004;39(3):746-753. 